\title{
A SIMPLIFIED SUBOCCIPITAL TECHNIQUE FOR TRIGEMINAL, ACOUSTIC, OR GLOSSOPHARYNGEAL RHIZOTOMY
}

\author{
BY
}

\author{
EXUM WALKER
}

Atlanta, Georgia

A new technique for the suboccipital approach to the cerebello-pontine angle was presented to this society* in November, 1941, but because of the advent of war, publication was postponed. This technique was developed principally for section of the trigeminal root.for tic douloureux, but is useful for certain other procedures in the angle.

The present report is based on our experience with this method in about 175 cases. This approach has prowed in my experience to be easier to carry out than the temporal approach. Furthermore, the clinical result has been superior in that subjective numbness is considerably less and the corneal reflex can almost always be preserved.

Although the technique is simple, observance of several essential points is required; otherwise, the procedure may prove to be difficult or even hazardous.

The patient is placed on his side and the head of the table is elevated well above the lumbar region. The patient's head is flexed well forward to facilitate access to the sub-occipital region. A lumbar spinal puncture is performed, but no fluid is released. An incision is made 4 to $5 \mathrm{~cm}$. in length and is placed vertically with its mid-point about $5 \mathrm{~cm}$. posterior to the external auditory canal. This is carried to the bone and an adequate area of bone is exposed with a periosteal elevator. A small, self-retaining retractor will usually control the bleeding, although electro-coagulation may be necessary. Occasionally the mastoid emissary vein may be torn, but can be controlled easily by electrocoagulation and bone wax.

Although I perform the procedure through a burr hole one inch in diameter, a larger opening is recommended until the surgeon becomes accustomed to the procedure. If a small opening is used, a headlight which provides light between or near the operator's eyes is essential. The burr hole is placed so that its upper margin includes the lambdoid suture and the lateral margin includes the occipito-

* Presented at the meeting of the American Academy of Neurosurgery in Montreal, Canada, September 22, 1948. mastoid suture. This should expose the transverse sinus both superiorly and laterally. A V-shaped incision is made in the dura carrying each limb laterally to the sinus and reflected on a base formed by the sinus.

At this stage spinal fluid is allowed to drain from the lumbar puncture needle. Drainage will allow the cerebellum to fall away from the petrous ridge by gravity, and the space will be void of fluid. The approach is along the petrous ridge adjacent to the tentorial attachment. Occasionally a petrosal vein will have to be coagulated and divided. The eighth nerve is brought into view and the arachnoid torn just cephalad to it. This brings the sensory root of the fifth nerve into view about $1 \mathrm{~cm}$. deeper. A small blade retractor is used to retract the pons gently ; this keeps the root taut and thus facilitates its division. The caudal portion of the nerve is divided by a flexible hook at its juncture with the pons. This is usually bloodless, but the patient must be well anæsthetized before the root is manipulated or divided as otherwise he may move or strain. Straining may cause the angle to become filled with fluid, and this will obscure vision and endanger the safety of structures deep in the angle.

After the root is inspected to be sure that the intended portion has been divided, the spinal needle is removed, the angle filled with saline, and the wound closed. For third division pain about onethird of the nerve is divided ; for second division pain, one half ; and for first division pain, about three-fifths.

While the procedure is simple, it requires meticulous planning and attention to detail and gentle caution during the intracranial part. Bleeding from the nerve root will be minimal or absent, provided that the division is made adjacent to the pons.

If bleeding occurs into the cerebello-pontine angle, an attempt should not be made to pursue the source of bleeding. Bleeding will usually stop if the angle is packed with cotton superficial to the eighth nerve, and time for clotting is allowed. In only one instance has it been necessary to close the wound 

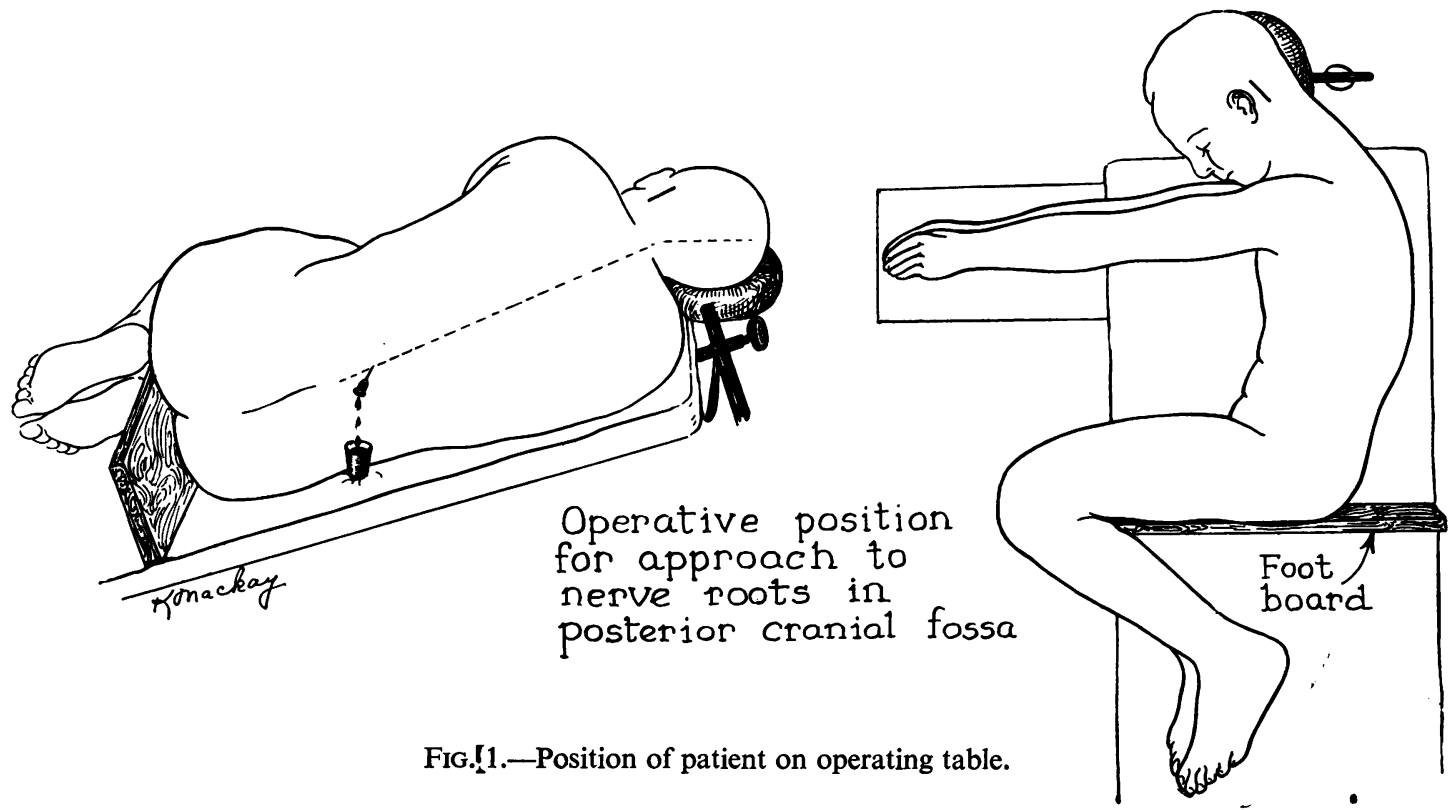

FIG.!1.-Position of patient on operating table.

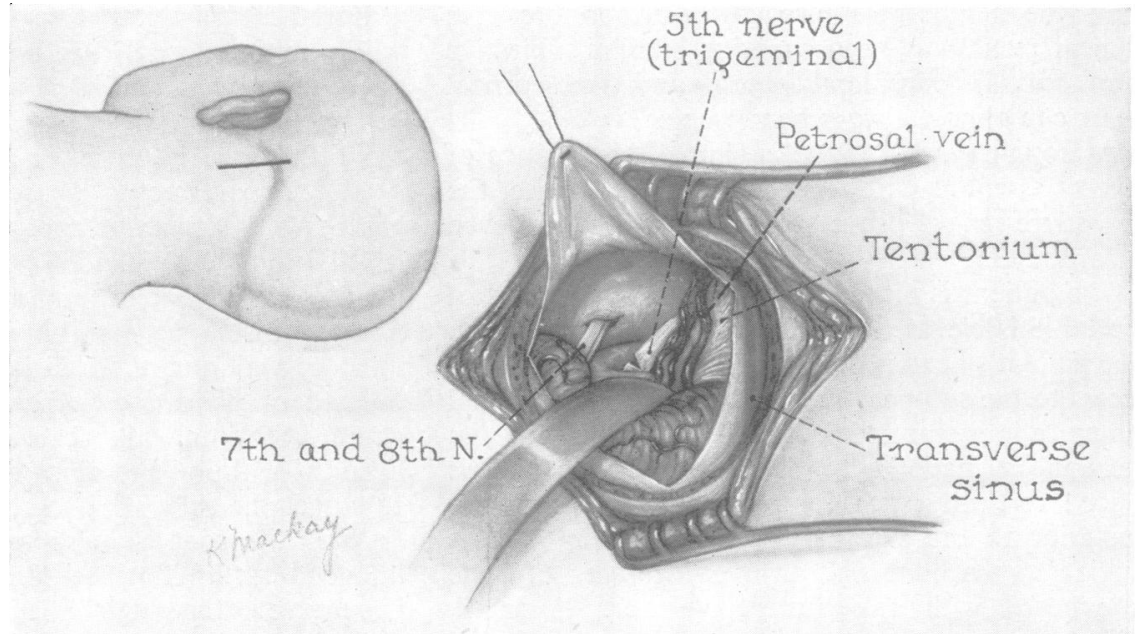

Fig. 2.-Site of intive exposure.

because of bleeding without dividing the nerve, but within a few days the wound was reopened and the nerve divided without difficulty.

The mastoid cells are often entered. If this occurs, they should be filled with bone wax and the dura closed carefully. In two instances rhinorrhœa developed which necessitated reopening of the incision and blocking the flow of fluid through the dura and into the mastoid cells.

On two occasions decerebrate rigidity developed during the operation. This subsided promptly after the stylet was replaced in the spinal needle and the angle filled with saline : it was thought to be due

to sagging of the mid brain against the tentorial edge. It is now my practice to replace the stylet after the fluid has drained out of the angle, removing it again whenever necessary.

No infections have occurred, and there has been but one fatality. This was in an eighty-six-year-old woman who was grateful for the relief of pain but wished to die. She had become ambulatory but would not leave hospital. She returned to bed and refused to eat, dying 22 days after operation.

In three instances, all of the pain was not relieved. Two of these cases were reoperated upon and the pain relieved by dividing a few additional fibres. 
(The motor root is always preserved because of its cephalad position.) There have been no instances of corneal ulcerations, and no precautions have been used to protect the eye even in the few cases where the corneal reflex was lost. Herpes about the lips have been rare. No patients have complained of paræsthesias.

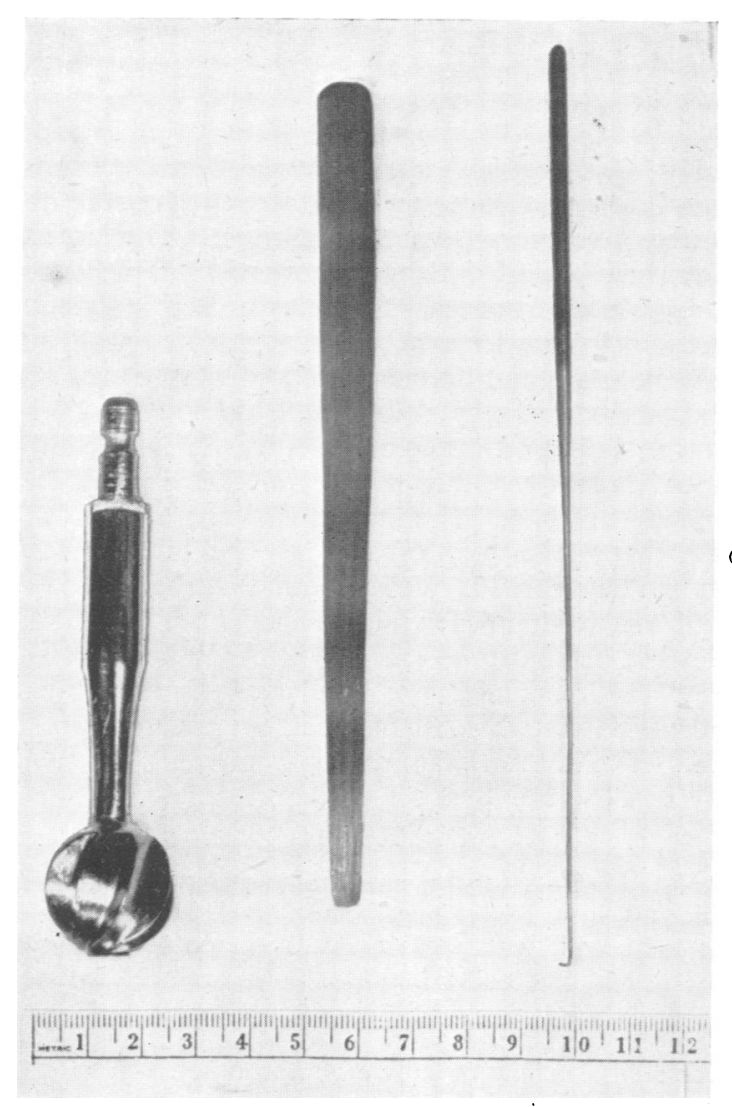

FIG. 3.-Special instruments used which facilitate operative procedure.

After this operation sensory loss was much less in the average case when compared to that which results after trigeminal rhizotomy by the temporal approach. When only one-third of the root is divided, the patient is often unaware of any subjective sensory loss. When tested objectively, there is hypæsthesia and hypalgesia, which is usually greatest in the third division distribution, but may be greatest in the second division area. It is always least in the first division. The loss becomes progressively increased as more fibres are divided, but is seldom complete in any area unless the root is almost totally divided. This preservation of sensation compared with the resultant total loss of sensation when the root is divided near the ganglion is probably due to rearrangement of the sensory fibres as the pons is approached.

My clinical experience suggests that it is not necessary to divide all of the fibres from any involved division of the nerve but only enough to reduce the afferent impulses to the point where the attacks of pain are no longer initiated.

In one instance division of about one-fifth of the nerve was sufficient to stop pain in all three divisions with no recurrence after ten years, and the patient had no demonstrable sensory loss. A total ganglionectomy on the opposite side had been performed 12 years previously.

The three patients in whom the pain was not relieved proves that a greater division of the nerve may be necessary in some cases. In younger patients I have often gambled and divided only onefourth to one-third of the nerve when two or three divisions were involved. This has usually relieved the pain with practically no subjective sensory loss.

It was this relative preservation of sensation which encouraged me to persist in working out the technique for this procedure despite some trying moments during my earlier experiences. I now feel that it is not only a more desirable procedure for the patient but is technically an easier operation than the temporal approach. It has been a practical solution to the patients who had previously had a rhizotomy of the opposite side by the temporal approach. These patients were assured of preservation of the motor function and of sufficient sensation to avoid difficulty in eating.

The procedure also offers access to the other nerve roots in the angle so that division of the acoustic or glossopharyngeal roots may be carried out easily. This has simplified the problem of division of the fifth and ninth roots at the same session. I have often employed this approach for diagnostic visualization of the angle. The opening can easily be enlarged if a tumour or other lesion is encountered.

\section{Summary}

An operative technique for the suboccipital approach to the cerebellopontine angle is described. Although developed for trigeminal rhizotomy, it is equally useful for acoustic or glossopharyngeal rhizotomy and for diagnostic visualization of the angle. The hazards and complications are discussed and some advantages over the temporal approach for tic cases are pointed out. 